\title{
Focused Ion Beams for Imaging, Analysis, and Fabrication - Where Did They Come From and Where are They Going?
}

\author{
John Notte ${ }^{1}$ \\ 1. Carl Zeiss Microscopy, Ion Microscopy Innovation Center, Peabody, MA 01960, USA
}

In his famous 1959 lecture, "There is Plenty of Room at the Bottom"[1], Feynman appealed to charged particle beams to help us see and manipulate matter at the smallest scales. At present, Focused Ion Beam (FIB) instruments represent a much smaller segment of the broader family of focused charge particle beam instruments (i.e. inclusive of SEMs and TEMs). And while FIBs were developed after electron beams, and have matured relatively slowly, the present situation can be described as a FIB renaissance, with many new instrument types and new applications coming to fruition. This paper provides a review of the FIB history, and an assessment of the most recent technical developments. This paper also considers the unique physics of ion-sample interaction and consequentially the applications which are enabled.

The primary quality of a FIB, the size of the focused probe for a given probe current, is mostly limited by the brightness of the ion source. (Unlike most SEMs, diffraction effects do not appreciably limit the FIB's focused probe size.) As such, the advancements in FIB capabilities follow closely the advancements of the ion source technologies. The gas field ion source (GFIS) is the brightest of the ion sources, and was developed first in 1955 [2], but progress was slow and was practically abandoned in the 1980's when the gallium liquid metal ion source (LMIS) became commercially available. For the last three decades the gallium LMIS has been optimized until it is now widely recognized as the workhorse and the most ubiquitous member of the FIB family. Yet there remain unmet application needs which the gallium FIB cannot address, and these have driven advancement of alternative FIB technologies. In the recent years, we have seen significant advancement and even commercial success in other technologies [3]: The GFIS technology has been reborn with both helium and neon species available with a sub nanometer probe sizes. At the same time, the plasma ion source has become increasingly valued, not for a small probe size, but for its very high probe currents which provide for high rates of material removal. And most recently, a laser based ion source has been developed which provides an extremely low temperature (monochromatic) ion beam with a selection of viable ion species. Figure 1 shows a rough timeline of the various FIB manufacturers in the last 30 years.

The utility of each FIB instrument is based upon the ion's unique beam-sample interaction. Any ion species will have a much higher mass than the electron, and correspondingly, for typical beam energies (Fig 2), the ion beam will have a much lower velocity and hence interact with matter in a fundamentally different manner than electron beams. The variety of ion species now available further broadens the range of applications from high resolution imaging with light ions such as helium (Fig 3) to large scale sputtering with high mass, high current ion sources, and even nanofabrication with newly developed FIBs.

\section{References:}

[1] Richard P. Feynman, Caltech Engineering and Science, 23:5, Feb. (1960), pp 22-36.

[2] Allan J. Melmed, Applied Surface Science, 94/95 (1996), pp 17-25. 
[3] Noel S. Smith, John A. Notte, Adam V. Steel, MRS Bulletin 39, (2014), pp 329-335.

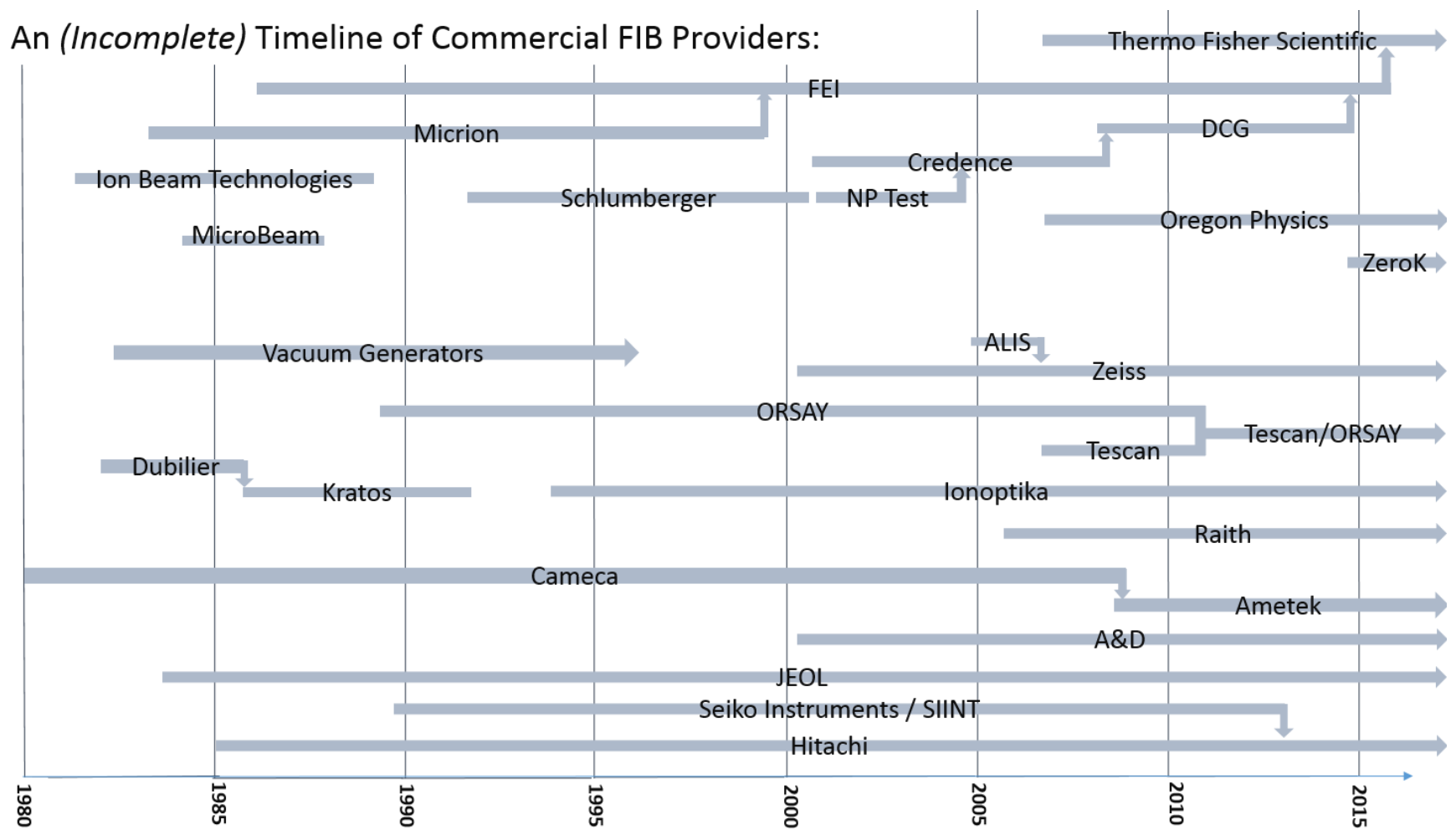

Figure 1. An assembled timeline of several of the commercial providers of FIBs and FIB instruments.

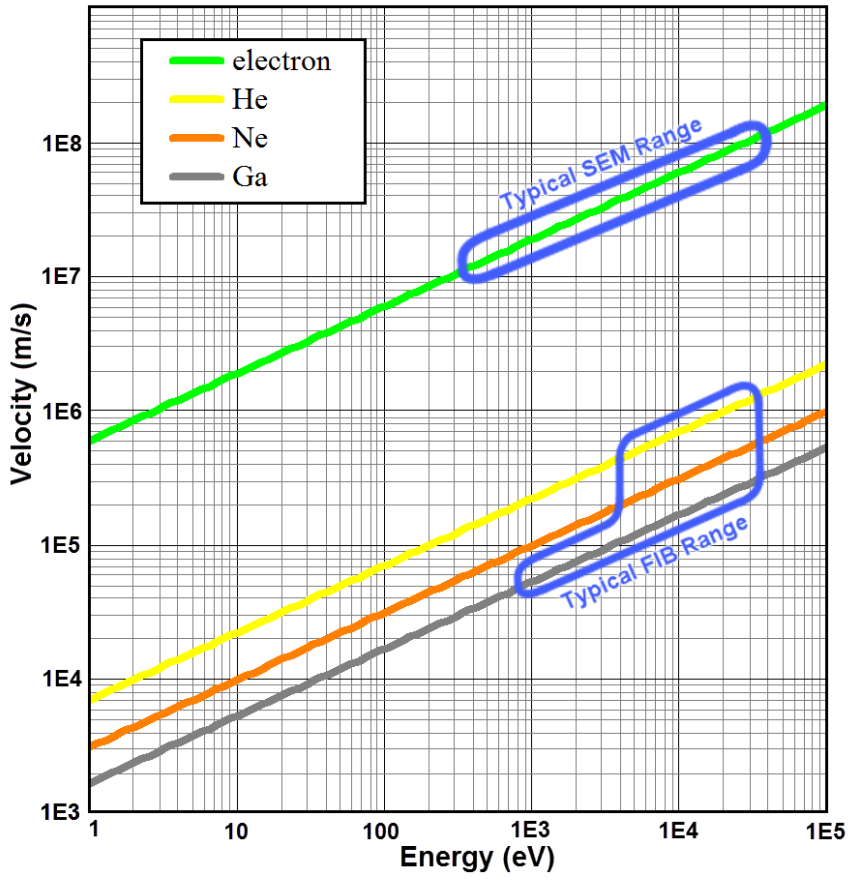

Figure 2. The velocities of various charged particles over their typical operating beam energies.

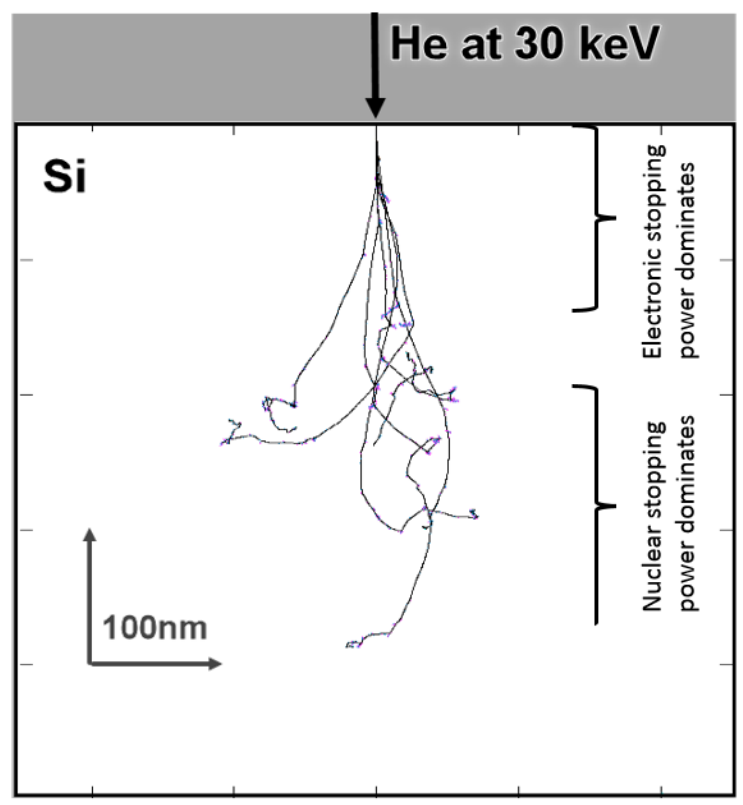

Figure 3. A SRIM simulation of helium ions entering a silicon sample. 